\title{
ON THE RESIDUAL FINITENESS AND OTHER PROPERTIES OF (RELATIVE) ONE-RELATOR GROUPS
}

\author{
STEPHEN J. PRIDE
}

(Communicated by Jonathan I. Hall)

\begin{abstract}
A relative one-relator presentation has the form $\mathcal{P}=\langle\mathbf{x}, H ; R\rangle$ where $\mathbf{x}$ is a set, $H$ is a group, and $R$ is a word on $\mathbf{x}^{ \pm 1} \cup H$. We show that if the word on $\mathbf{x}^{ \pm 1}$ obtained from $R$ by deleting all the terms from $H$ has what we call the unique max-min property, then the group defined by $\mathcal{P}$ is residually finite if and only if $H$ is residually finite (Theorem 1 ). We apply this to obtain new results concerning the residual finiteness of (ordinary) one-relator groups (Theorem 4). We also obtain results concerning the conjugacy problem for onerelator groups (Theorem 5), and results concerning the relative asphericity of presentations of the form $\mathcal{P}$ (Theorem 6).
\end{abstract}

\section{INTRODUCTION}

The question of when one-relator groups are residually finite is still open.

In the torsion-free case there are well-known examples of groups which are not residually finite, namely the Baumslag-Solitar/Meskin groups [4], [15]:

$$
G=\left\langle\mathbf{x} ; U^{-1} V^{l} U V^{m}\right\rangle,
$$

where $U, V$ do not generate a cyclic subgroup of the free group on $\mathbf{x}$, and $|l| \neq|m|$, $|l|,|m|>1$. On the other hand, there are some examples which are known to be residually finite. For instance, it was shown in [3] that if

$$
W=U V^{-1} \text {, }
$$

where $U, V$ are positive words on an alphabet $\mathbf{x}$ and the exponent sum of $x$ in $U V^{-1}$ is 0 for each $x \in \mathbf{x}$, or if

$$
W=[U, V],
$$

where $U, V$ are (not necessarily positive) words on $\mathbf{x}$ such that no letter $x \in \mathbf{x}$ appears in both $U$ and $V$, then $G=\langle\mathbf{x} ; W\rangle$ is residually finite.

In the torsion case there is the well-known open question:

Question 1 ([2], [5, Question OR1]). Is every one-relator group with torsion residually finite?

Received by the editors June 5, 2006.

2000 Mathematics Subject Classification. Primary 20E26, 20F05; Secondary 20F10, 57M07.

Key words and phrases. Residual finiteness, one-relator group, relative presentation, (power) conjugacy problem, asphericity, unique max-min property, 2-complex of groups, covering complex. 
Question 1 is known to be true when $G=\left\langle\mathbf{x} ; W^{n}\right\rangle$ where $W$ is a positive word and $n>1$ [9] (see also [19]). In [20], Wise obtains further related results, summed up by his "Quasi-Theorem 1.3": If $W$ is sufficiently positive, and $W^{n}$ is sufficiently small cancellation, then $G$ is residually finite.

A related open question is:

Question 2 ([5, Question OR6], [11, Question 8.68]). If a torsion-free one-relator group $G_{1}=\langle\mathbf{x} ; W\rangle$ is residually finite, then is $G_{n}=\left\langle\mathbf{x} ; W^{n}\right\rangle$ also residually finite for $n>1$ ?

(Of course, if Question 1 is true, then Question 2 is trivially true.)

It was shown in [1] that Question 2 holds true when $W$ has the form (1) or (2).

Here, amongst other things, we tackle Question 2 by considering relative presentations.

A relative presentation has the form

$$
\mathcal{P}=\langle\mathbf{x}, H ; \mathbf{r}\rangle,
$$

where $H$ is a group and $\mathbf{r}$ is a set of expressions of the form

$$
R=x_{1}^{\varepsilon_{1}} h_{1} x_{2}^{\varepsilon_{2}} h_{2} \ldots x_{r}^{\varepsilon_{r}} h_{r}\left(r>0, x_{i} \in \mathbf{x}, \varepsilon_{i}= \pm 1, h_{i} \in H, 1 \leq i \leq r\right) .
$$

The word

$$
W=x_{1}^{\varepsilon_{1}} x_{2}^{\varepsilon_{2}} \ldots x_{r}^{\varepsilon_{r}} \quad\left(r>0, x_{i} \in \mathbf{x}, \varepsilon_{i}= \pm 1,1 \leq i \leq r\right)
$$

is called the $\mathbf{x}$-skeleton of $R$. We do not require that the $\mathbf{x}$-skeleton be reduced or cyclically reduced. The group $G=G(\mathcal{P})$ defined by $\mathcal{P}$ is the quotient of $H * F$ (where $F$ is the free group on $\mathbf{x}$ ) by the normal closure of the elements of $H * F$ represented by the expressions $R \in \mathbf{r}$. The composition of the canonical imbedding $H \rightarrow H * F$ with the quotient map $H * F \rightarrow G$ is called the natural homomorphism, denoted by $\nu: H \rightarrow G$ (or simply $H \rightarrow G$ ).

As is normal, we will often abuse notation and write $G=\langle\mathbf{x}, H ; \mathbf{r}\rangle$, or $G \cong$ $\langle\mathbf{x}, H ; \mathbf{r}\rangle$.

When $\mathbf{r}$ consists of a single element $R$, then we have the one-relator relative presentation

$$
\mathcal{P}=\langle\mathbf{x}, H ; R\rangle .
$$

Heuristically, $G=G(\mathcal{P})$ should be governed by the shape of the x-skeleton of $R$ and the algebraic properties of $H$.

Here we introduce the unique max-min property for the shape of $W$. (Words of the form (1) are a very special case.) For a group $H$, denote by $\mathcal{M}_{H}$ the class of one-relator relative presentations of the form (5), where $W$ has the unique max-min property.

Theorem 1. If $P$ is in $\mathcal{M}_{H}$, then

(i) the natural homomorphism $H \rightarrow G(\mathcal{P})$ is injective;

(ii) $G(\mathcal{P})$ is residually finite if and only if $H$ is residually finite.

We can deduce from this

Theorem 2 (Substitution theorem). Let $K$ be a one-relator group given by an ordinary presentation $\langle\mathbf{y}, z ; S(\mathbf{y}, z)\rangle$, and let $\mathcal{P}=\langle\mathbf{x}, H ; R\rangle$ be an $\mathcal{M}_{H}$-presentation. Then the group given by the relative presentation $\langle\mathbf{x}, \mathbf{y}, H ; S(\mathbf{y}, R)\rangle$ is residually finite if and only if $H$ and $K$ are residually finite. 
We can give the proof of this straightaway. Consider the $\mathcal{M}_{H * K}$-presentation $\overline{\mathcal{P}}=\left\langle\mathbf{x}, H * K ; R z^{-1}\right\rangle$. By Theorem $1, L=G(\overline{\mathcal{P}})$ is residually finite if and only if $H * K$ is residually finite, which is equivalent to requiring that both $H$ and $K$ are residually finite (using results discussed in [12], p. 417). Now note that

$$
L \cong\left\langle\mathbf{x}, \mathbf{y}, z, H ; S(\mathbf{y}, z), R z^{-1}\right\rangle \cong\langle\mathbf{x}, \mathbf{y}, H ; S(\mathbf{y}, R)\rangle
$$

In particular, taking $K$ to be defined by $\left\langle z ; z^{n}\right\rangle(n>1)$ we have

Theorem 3. If $G=\langle\mathbf{x}, H ; R\rangle$ is a residually finite $\mathcal{M}_{H}$-group, then the group $G_{n}=\left\langle\mathbf{x}, H ; R^{n}\right\rangle(n>1)$ is also residually finite.

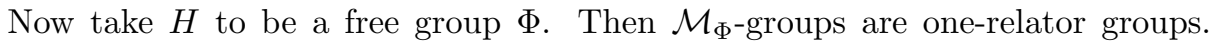
Since $\Phi$ is residually finite ([12], p. 116 or p. 417), we obtain the following theorem concerning the residual finiteness of one-relator groups.

Theorem 4. Every $\mathcal{M}_{\Phi-\text { group }} G=\langle\mathbf{x}, \Phi ; R\rangle$ is a residually finite one-relator group. Moreover, if $K=\langle\mathbf{y}, z ; S(\mathbf{y}, z)\rangle$ is a one-relator group, then the one-relator group $\bar{K}=\langle\mathbf{x}, \mathbf{y}, \Phi ; S(\mathbf{y}, R)\rangle$ is residually finite if and only if $K$ is residually finite. In particular, $G_{n}=\left\langle\mathbf{x}, \Phi ; R^{n}\right\rangle(n>1)$ is residually finite.

The solution of the conjugacy problem for one-relator groups with torsion has been solved by B. B. Newman [16]. However, for the torsion-free case the problem is still open [5, Question O5].

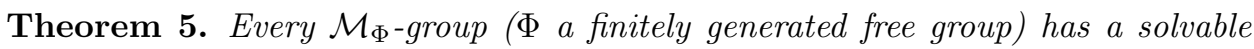
conjugacy problem. Also, such groups have a solvable power conjugacy problem.

(Two elements $c, d$ of a group are said to be power conjugate if some power of $c$ is conjugate to some power of $d$.)

Other aspects of relative presentations (and in particular, one-relator relative presentations) have been studied intensively, particularly asphericity. Recall [6] that a relative presentation $\mathcal{P}$ is aspherical (more accurately, diagrammatically aspherical) if every spherical picture over $\mathcal{P}$ contains a dipole. Under a weaker condition on shape (the unique min property, or equivalently the unique max property) we can prove

Theorem 6. Let $\mathcal{P}$ be a relative presentation as in (5), where $W$ has the unique min property. Then $\mathcal{P}$ is aspherical.

It then follows from [6] (see Corollary 1 of Theorem 1.1, Theorem 1.3, and Theorem 1.4) that for the group $G=G(\mathcal{P})$ we have

(i) the natural homomorphism $H \rightarrow G$ is injective;

(ii) every finite subgroup of $G$ is contained in a conjugate of $H$;

(iii) for any left $\mathbb{Z} G$-module $A$ and any right $\mathbb{Z} G$-module $B$,

$$
\begin{aligned}
& H^{n}(G, A) \cong H^{n}(H, A), \\
& H_{n}(G, B) \cong H_{n}(H, B)
\end{aligned}
$$

for all $n \geq 3$. 


\section{MAX-MIN PROPERTY}

Let $\mathbf{x}$ be an alphabet. A weight function on $\mathbf{x}$ is a function

$$
\theta: \mathbf{x} \longrightarrow \mathbb{Z}
$$

such that $\operatorname{Im} \theta$ generates the additive group $\mathbb{Z}$ (that is, $\operatorname{gcd}\{\theta(x): x \in \mathbf{x}\}$ is 1 ). A strict weight function is one for which $\theta(x) \neq 0$ for all $x \in \mathbf{x}$.

Let $W$ be a word on $\mathbf{x}$ as in (4). Given a weight function $\theta$, we then have the function

$$
\begin{aligned}
\phi & =\phi_{W}^{\theta}:\{0,1,2, \ldots, r\} \rightarrow \mathbb{Z}, \\
\phi(j) & =\sum_{i=0}^{j} \varepsilon_{i} \theta\left(x_{i}\right),
\end{aligned}
$$

where $\phi(0)=0$ since the empty sum is taken to be 0 . We will say that the weight function is admissible for $W$ if $\phi(r)=0$.

For visual purposes, it is useful to extend $\phi$ to a piecewise linear function $\phi$ : $[0, r] \rightarrow \mathbb{R}$, so that the graph of $\phi$ in the interval $[j-1, j]$ is the straight line segment joining the points $(j-1, \phi(j-1)),(j, \phi(j))(0<j \leq r)$. We will informally refer to this graph as "the graph of $W$ " (with respect to $\theta$ ).

A word $W$ as in (4) will be said to have the unique max-min property if for some admissible strict weight function $\theta$, the graph of $W$ has a unique maximum and a unique minimum. To be precise, we require that, for some admissible strict weight function and some $k, l \in\{1,2, \ldots, r\}$, we have $\phi(j)<\phi(k)$ for all $j \in$ $\{1,2, \ldots, r\}-k$ and $\phi(j)>\phi(l)$ for all $j \in\{1,2, \ldots, r\}-\{l\}$. We also require that $x_{k} \neq x_{k+1}$ and $x_{l} \neq x_{l+1}$ (subscripts modulo $r$ ). This amounts to requiring that $W$ is "reduced at the unique maximum and minimum"; that is, $x_{k}^{\varepsilon_{k}} \neq x_{k+1}^{-\varepsilon_{k+1}}, x_{l}^{\varepsilon_{l}} \neq$ $x_{l+1}^{-\varepsilon_{l+1}}$ (subscripts modulo $r$ ). For at the maximum and minimum we must have either $x_{j} \neq x_{j+1}$, or $x_{j}=x_{j+1}$ and $\varepsilon_{j}=-\varepsilon_{j+1}(j=k, l)$. If the two letters occurring at the unique maximum are not disjoint from the two letters occurring at the unique minimum (i.e. $\left\{x_{k}, x_{k+1}\right\} \cap\left\{x_{l}, x_{l+1}\right\}$ is not empty), then we will say that $W$ has the strong unique max-min property.

A word $W$ as in (4) will be said to have the unique min property if for some strict weight function $\theta$, the graph of $W$ has a unique minimum (but not necessarily a unique maximum). The unique max property is defined similarly, but is not really of interest because replacing $\theta$ by $-\theta$ will convert this property to the unique min property.

We let $\mathcal{M}_{H}^{1}$ (respectively $\mathcal{S}_{H}^{1}$ ) denote the subclass of $\mathcal{M}_{H}$ consisting of relative presentations of the form (5) for which $W$ has the unique max-min property (respectively, the strong unique max-min property) with respect to the weight function

$$
\mathbf{1}: \mathbf{x} \longrightarrow \mathbb{Z} \quad x \mapsto 1(x \in \mathbf{x}) .
$$

Lemma 1. Every $\mathcal{M}_{H^{-}}$group can be embedded into an $\mathcal{M}_{H^{-}}^{1}$ group.

Proof. Let $G=\langle\mathbf{x}, H ; R\rangle$ with $R$ as in (3), and suppose $W=x_{1}^{\varepsilon_{1}} x_{2}^{\varepsilon_{1}} \ldots x_{r}^{\varepsilon_{r}}$ has the unique max-min property with respect to some strict weight function $\theta: \mathbf{x} \rightarrow \mathbb{Z}$. We can assume $\theta(x)>0$ for all $x$. For if $\theta(x)<0$, then we can replace $x$ by $x^{-1}$.

Let

$$
\mathbf{y}=\{y: y \in \mathbf{x}, \theta(y)>1\},
$$


and let

$$
\hat{\mathbf{x}}=(\mathbf{x}-\mathbf{y}) \cup\left\{y_{1}, y_{2}, \ldots, y_{\theta(y)}: y \in \mathbf{y}\right\} .
$$

Let $\hat{G}=\langle\hat{\mathbf{x}}, H ; \hat{R}\rangle$, where $\hat{R}$ is obtained from $R$ by replacing each occurrence of $y^{ \pm 1}$ by $\left(y_{1} y_{2} \ldots y_{\theta(y)}\right)^{ \pm 1}(y \in \mathbf{y})$. It is easy to see that the $\hat{\mathbf{x}}$-skeleton $\hat{W}$ of $\hat{R}$ has the unique max-min property with respect to $\mathbf{1}: \hat{\mathbf{x}} \rightarrow \mathbb{Z}$. (The graph of $\hat{W}$ is obtained from that of $W$ by stretching along the horizontal axis.) Moreover, $G$ is embedded into $\hat{G}$, for we have the retraction $\rho$ with section $\mu$ :

$$
\begin{gathered}
\hat{G} \stackrel{\rho}{\rightleftarrows} \underset{\mu}{\rightleftarrows} \quad \rho \mu=\operatorname{id}_{G}, \\
\rho: x \mapsto x(x \in \mathbf{x}-\mathbf{y}), y_{1} \mapsto y, y_{i} \mapsto 1(y \in \mathbf{y}, 1<i \leq \theta(y)), h \mapsto h(h \in H), \\
\mu: x \mapsto x(x \in \mathbf{x}-\mathbf{y}), y \mapsto y_{1} y_{2} \ldots y_{\theta(y)}(y \in \mathbf{y}), h \mapsto h(h \in H) .
\end{gathered}
$$

Lemma 2. Every $\mathcal{M}_{H^{1}}^{\mathbf{1}}$ group can be embedded into an $\mathcal{S}_{H^{1}}^{\mathbf{1}}$ group.

Proof. Let $G=\langle\mathbf{x}, H ; R\rangle$, where the x-skeleton $W$ of $R$ has the unique maxmin property with respect to the constant function $\mathbf{1}: \mathbf{x} \rightarrow \mathbb{Z}$. Suppose the letters occurring at the unique maximum are $a, b$, and those occurring at the unique minimum are $c, d$. We can assume that $\{a, b\} \cap\{c, d\}$ is empty; otherwise, there is nothing to prove. Let $\mathbf{y}=\mathbf{x}-\{a, b, c, d\}$, and introduce a new alphabet

$$
\hat{\mathbf{x}}=\{a, b, c, d, e\} \cup\left\{y_{1}, y_{2}: y \in \mathbf{y}\right\} .
$$

Let $\hat{R}$ be obtained from $R$ as follows. For each $y \in \mathbf{y}$, replace all occurrences of $y^{ \pm 1}$ by $\left(y_{1} y_{2}\right)^{ \pm 1}$, and replace all occurrences of $a^{ \pm 1}$ (respectively, $b^{ \pm 1}, c^{ \pm 1}, d^{ \pm 1}$ ) by $(e a)^{ \pm 1}$ (respectively, $(b e)^{ \pm 1},(e c)^{ \pm 1},(d e)^{ \pm 1}$ ). Let $\hat{G}=\langle\hat{\mathbf{x}}, H ; \hat{R}\rangle$, and let $\hat{W}$ be the word obtained from $\hat{R}$ by deleting all terms from $H$. The graph of $\hat{W}$ under the weight function $\mathbf{1}: \hat{\mathbf{x}} \rightarrow \mathbb{Z}$ is the graph of $W$ magnified by a factor of 2 , and $e$ occurs at the unique maximum and the unique minimum. Moreover, $G$ is embedded into $\hat{G}$ for we have the retraction $\rho$ with section $\mu$ :

$$
\begin{gathered}
\hat{G} \stackrel{\rho}{\rightleftarrows} G \quad \rho \mu=\operatorname{id}_{G}, \\
\rho: z \mapsto z(z \in\{a, b, c, d\}), e \mapsto 1, y_{1} \mapsto y, y_{2} \mapsto 1(y \in \mathbf{y}), h \mapsto h(h \in H), \\
\mu: a \mapsto e a, b \mapsto b e, c \mapsto e c, d \mapsto d e, y \mapsto y_{1} y_{2}(y \in \mathbf{y}), h \mapsto h(h \in H) .
\end{gathered}
$$

Remark 1. Note that in both the above proofs, we have $\mu \nu=\hat{\nu}$, where $\nu: H \rightarrow G$, $\hat{\nu}: H \rightarrow \hat{G}$ are the natural homomorphims. Thus if $\hat{\nu}$ is injective, then so is $\nu$.

Remark 2. Note also from the proof of the above two lemmas, we get that every $\mathcal{M}_{H}$-group is a retract of an $\mathcal{S}_{H}^{1}$-group.

Remark 3. The referee has brought to my attention the work of K. S. Brown [8], which is concerned with whether a homomorphism $\chi$ from a one-relator group $B=\langle\mathbf{x} ; W\rangle(|\mathbf{x}| \geq 2, W$ as in (4) and cyclically reduced) onto $\mathbb{Z}$ has a finitely generated kernel. Such a homomorphism is induced by a weight function $\theta$ which is admissible for $W$. However, since $\theta$ need not be strict, it is necessary to interpret the max-min property more widely. Thus the unique maximum could be a plateau: i.e., for some $k \in\{1,2, \ldots, r\}$ we could have $\phi(k)=\phi(k+1)$ and $\phi(j)<\phi(k)$ for all $j \in\{1,2, \ldots, r\}-\{k, k+1\}$ (subscripts modulo $r$ ). Similarly, the unique minimum could be a reverse plateau. Then according to Brown [8], as restated in Theorem 2.2 
of [13], ker $\chi$ is finitely generated if and only if $|\mathbf{x}|=2$, and $W$ has the unique maxmin property in the above sense with respect to the corresponding weight function. In our work we could also allow non-strict weight functions. However, for the most part this can be avoided. For example, if the unique maximum is a plateau with $x_{k} \neq x_{k+2}$, then we could transform it to a genuine maximum by deleting $x_{k+1}$ from $\mathbf{x}$ and replacing $H$ by $H *\left\langle x_{k+1}\right\rangle$. However, if the unique maximum is a plateau with $x_{k}=x_{k+2}$, then some of our arguments need to be modified, which we leave as an exercise for the reader.

\section{A CONSTRUCtion}

By a 2-complex of groups we mean a connected graph of groups (in the sense of Serre [18]) with trivial edge groups, together with a set of closed paths which we call defining paths. (These are essentially the generalized complexes defined in $\S 1$ of [10], where more detail can be found. Note however, that in [10] a 2-cell $c(\alpha)$ consists of all cyclic permutations of $\alpha^{ \pm 1}$ for each one of our defining paths $\alpha$. We specifically do not add these extra paths. This makes no significant difference.)

Let $\mathcal{P}$ be as in (5), and let $\theta$ be an admissible weight function for $W$. There is then an induced epimorphism

$$
\psi: G \rightarrow \mathbb{Z} \quad x \mapsto \theta(x)(x \in \mathbf{x}), h \mapsto 0(h \in H) .
$$

We can construct a 2-complex of groups

$$
\tilde{\mathcal{P}}=\left\langle\Gamma, H_{n}(n \in \mathbb{Z}) ;(n, R)(n \in \mathbb{Z})\right\rangle
$$

whose fundamental group is isomorphic to the kernel $K$ of $\psi$. The underlying graph $\Gamma$ has vertex set $\mathbb{Z}$, edges $\left(n, x^{\varepsilon}\right)(n \in \mathbb{Z}, x \in \mathbf{x}, \varepsilon= \pm 1)$, and initial, terminal and inversion functions $\iota, \tau,{ }^{-1}$ given by $\iota\left(n, x^{\varepsilon}\right)=n, \tau\left(n, x^{\varepsilon}\right)=n+\varepsilon \theta(x),\left(n, x^{\varepsilon}\right)^{-1}=$ $\left(n+\varepsilon \theta(x), x^{-\varepsilon}\right)$. The vertex groups are copies $H_{n}=\{(n, h): h \in H\}$ of $H$ (with the obvious multiplication $\left.(n, h)\left(n, h^{\prime}\right)=\left(n, h h^{\prime}\right)\right)$. We extend $\iota, \tau,^{-1}$ to the elements of the vertex groups by defining $\iota(n, h)=n=\tau(n, h),(n, h)^{-1}=\left(n, h^{-1}\right)$, where $h^{-1}$ is the inverse of $h$ in $H$. We extend $\theta$ to $\mathbf{x}^{ \pm 1} \cup H$ by defining $\theta\left(x^{-1}\right)=-\theta(x)$ $(x \in \mathbf{x}), \theta(h)=0(h \in H)$. Then for any sequence $\alpha=z_{1} z_{2} \ldots z_{q}$ with $z_{i} \in \mathbf{x}^{ \pm 1} \cup H$ and any vertex $n \in \Gamma$, we have a path $(n, \alpha)$ in the graph of groups starting at $n$, where

$$
\begin{aligned}
&(n, \alpha)=\left(n, z_{1}\right)\left(n+\theta\left(z_{1}\right), z_{2}\right)\left(n+\theta\left(z_{1}\right)\right.\left.+\theta\left(z_{2}\right), z_{3}\right) \\
& \ldots \\
&\left(n+\theta\left(z_{1}\right)+\theta\left(z_{2}\right)+\ldots+\theta\left(z_{q-1}\right), z_{q}\right) .
\end{aligned}
$$

In particular we have the (closed) paths $(n, R)$.

There is an obvious action of $\mathbb{Z}$ on the above graph of groups, with $i \in \mathbb{Z}$ acting on vertices by $i \cdot n=i+n(n \in \mathbb{Z})$, and on the edges and vertex groups by $i \cdot(n, z)=$ $(i+n, z)\left(n \in \mathbb{Z}, z \in \mathbf{x}^{ \pm 1} \cup H\right)$. This action of course extends to paths. Thus $(i, \alpha)=i .(0, \alpha)$. In particular, $(i, R)=i .(0, R)$, so $\mathbb{Z}$ acts on $\tilde{\mathcal{P}}$.

If we regard $\mathcal{P}$ as a 2-complex of groups with a single vertex $o$, edges $x^{\varepsilon}(x \in$ $\mathbf{x}, \varepsilon= \pm 1$ ), vertex group $H$, and defining path $R$, then we have a mapping of 2-complexes of groups

$$
\begin{gathered}
\rho: \tilde{\mathcal{P}} \longrightarrow \mathcal{P}, \\
n \mapsto o,\left(n, x^{\varepsilon}\right) \mapsto x^{\varepsilon},(n, h) \mapsto h,(n, R) \mapsto R
\end{gathered}
$$

$(n \in \mathbb{Z}, x \in \mathbf{x}, \varepsilon= \pm 1, h \in H)$. This induces a homomorphism

$$
\rho_{*}: \pi_{1}(\tilde{\mathcal{P}}, 0) \longrightarrow \pi_{1}(\mathcal{P}, o)=G
$$


which is injective, and $\operatorname{Im} \rho_{*}=K$. This can easily be proved by adapting the standard arguments of covering space theory for ordinary 2-complexes (see for example [17], pp. 157-159) to this relative situation.

\section{Proof of Theorem 1}

Since residual finiteness is closed under taking subgroups, it follows from Lemmas 1 and 2 and Remark 1 at the end of $\S 2$ that it suffices to prove Theorem 1 for $\mathcal{S}_{H^{-}}^{1}$ groups.

We will make use of the following results:

(a) A free product $F * B$, where $F$ is a free group, is residually finite if and only if $B$ is residually finite;

(b) An infinite cyclic extension of a finitely generated group $L$ is residually finite if and only if $L$ is residually finite.

(The first of these follows from results on p. 417 of [12]; the second is a special case of Theorem 7, p. 29 of [14].)

We can assume $\mathbf{x}$ is finite. For if not let $\mathbf{x}^{\prime}$ be the set of letters occurring in $R$. Then $G$ is isomorphic to $G^{\prime} * \Psi$ where $G^{\prime} \cong\left\langle\mathbf{x}^{\prime}, H ; R\right\rangle$, and $\Psi$ is the free group on $\mathbf{x}-\mathbf{x}^{\prime}$. So by (a) above, it is enough to work with $G^{\prime}$.

Let $G$ be defined by an $\mathcal{S}_{H}^{1}$ presentation as in (5), with $e \in \mathbf{x}$ occurring at both the unique maximum and the unique minimum of the graph of $W$ under the weight function $\theta=1$. We denote the maximum and minimum values of $\phi_{W}$ by $M, m$, respectively. Note that $m \leq 0 \leq M$ and $m<M$.

We first deal with the trivial case when $M-m=1$. Then up to cyclic permutation and inversion, $R=e h a^{-1} h^{\prime}$, where $a \in \mathbf{x}-\{e\}, h, h^{\prime} \in H$. Thus $G=\Phi * H$, where $\Phi$ is the free group on $\mathbf{x}-\{e\}$, so the theorem holds by (a) above.

Now suppose $M-m>1$. Let $f \in \mathbf{x}-\{e\}$.

We have the epimorphism

$$
\psi: G \rightarrow \mathbb{Z} \quad x \mapsto 1(x \in \mathbf{x}), h \mapsto 0(h \in H) .
$$

Also, we have the homomorphism

$$
\eta: \mathbb{Z} \rightarrow G \quad 1 \mapsto f
$$

Then $\psi \eta=\mathrm{id}_{\mathbb{Z}}$, so $G$ is a semidirect product $K \rtimes \mathbb{Z}$, where $K=\operatorname{ker} \psi$, and with the action of $n \in \mathbb{Z}$ on $K$ being induced by conjugation by $f^{n}$.

The fundamental group of $\tilde{\mathcal{P}}$ (at the vertex 0 ), as in $\S 3$, is isomorphic to $K$.

We will obtain a relative presentation for $K$ by collapsing a maximal tree.

The edges $(n, f)^{ \pm 1}$ form a maximal tree $T$ in $\Gamma$. Let $R_{n}$ be the word on $\{(i, x): i \in \mathbb{Z}, x \in \mathbf{x}, x \neq f\} \cup\left(\bigcup_{i \in \mathbb{Z}} H_{i}\right)$ obtained from $(n, R)$ by deleting all edges from $T$ which occur in $(n, R)$ and replacing all terms $\left(i, x^{-1}\right)$ by $(i-1, x)^{-1}(i \in$ $\mathbb{Z}, x \in \mathbf{x}, x \neq f)$. Then

$$
\mathcal{Q}=\left\langle(n, x)(n \in \mathbb{Z}, x \in \mathbf{x}, x \neq f), *_{n \in \mathbb{Z}} H_{n} ; R_{n}(n \in \mathbb{Z})\right\rangle
$$

is a relative presentation for $K$. Moreover, since the edges in $T$ constitute an orbit under the action of $\mathbb{Z}$ on our graph of groups, the action of $\mathbb{Z}$ on $K$ is given by the automorphism

$$
\mu:(n, x) \mapsto(n+1, x)(x \in \mathbf{x}, x \neq f),(n, h) \mapsto(n+1, h)(h \in H)
$$

$(n \in \mathbb{Z})$. 
Now consider the $H N N$-extension $\bar{K}$ of $K$ given by the relative presentation

$$
\begin{aligned}
\overline{\mathcal{Q}}= & \left\langle(n, x)(n \in \mathbb{Z}, x \in \mathbf{x}, x \neq f), *_{n \in \mathbb{Z}} H_{n}, s ; R_{n}(n \in \mathbb{Z})\right. \\
& s(n, x) s^{-1}=(n+1, x)(n \in \mathbb{Z}, x \in \mathbf{x}, x \neq e, f), \\
& \left.s(n, h) s^{-1}=(n+1, h)(n \in \mathbb{Z}, h \in H)\right\rangle .
\end{aligned}
$$

The automorphism $\mu$ of $K$ can be extended to an automorphism $\bar{\mu}$ of $\bar{K}$ by defining $\bar{\mu}(s)=s$. Then $G=K \rtimes_{\mu} \mathbb{Z}$ can be embedded into $\bar{G}=\bar{K} \rtimes_{\bar{\mu}} \mathbb{Z}$.

By our assumption, up to cyclic permutation and inversion, $(0, R)$ will have the form

$$
(M-1, e)(M, h)(M-1, a)^{-1} \gamma_{0}\left((m, b)^{-1}\left(m, h^{\prime}\right)(m, e)\right)^{\varepsilon} \delta_{0},
$$

where $h, h^{\prime} \in H, \varepsilon= \pm 1, a, b \in \mathbf{x}-\{e\}$, and each term $(i, z)$ occurring in the paths $\gamma_{0}, \delta_{0}$ is such that both its initial and terminal vertices lie in the range $m+1$, $m+2, \ldots, M-1$. Then

$$
R_{0}=(M-1, e) \alpha_{0}(m, e)^{\varepsilon} \beta_{0},
$$

where $\alpha_{0}, \beta_{0}$ do not contain any occurrence of $(i, e)^{ \pm 1}$ with $i \leq m$ or $i \geq M-1$. More generally, for $n \in \mathbb{Z}$

$$
R_{n}=(n+M-1, e) \alpha_{n}(n+m, e)^{\varepsilon} \beta_{n},
$$

where $\alpha_{n}, \beta_{n}$ do not contain any occurrence of $(i, e)^{ \pm 1}$ with $i \leq n+m$ or $i \geq$ $n+M-1$.

Let $F_{0}$ be the free group on

$$
(\mathbf{x}-\{e, f\}) \cup\{s,(m+1, e),(m+2, e), \ldots,(M-1, e)\} .
$$

Then there is a homomorphism

$$
\bar{K} \rightarrow H * F_{0}
$$

defined as follows:

$$
\begin{aligned}
& s \mapsto s, \\
& (n, x) \mapsto s^{n} x s^{-n}(x \in \mathbf{x}, x \neq e, f, n \in \mathbb{Z}), \\
& (n, h) \mapsto s^{n} h s^{-n}(h \in H, n \in \mathbb{Z}), \\
& (i, e) \mapsto(i, e) \quad(m+1 \leq i \leq M-1),
\end{aligned}
$$

and inductively, for $k=0,1,2, \ldots$,

$$
\begin{aligned}
(k+M, e) & \mapsto \beta_{k+1}^{-1}(k+1+m, e)^{-\varepsilon} \alpha_{k+1}^{-1}, \\
(-k+m, e) & \mapsto\left(\beta_{-k}(-k+M-1, e) \alpha_{-k}\right)^{-\varepsilon} .
\end{aligned}
$$

This homomorphism is actually an isomorphism. The inverse is defined by

$$
\begin{array}{ll}
x & \mapsto(0, x) \quad(x \in \mathbf{x}, x \neq e, f), \\
h & \mapsto(0, h) \quad(h \in H), \\
(i, e) & \mapsto(i, e) \quad m+1 \leq i \leq M-1, \\
s & \mapsto s .
\end{array}
$$

Thus $\bar{G}$ is an infinite cyclic extension of the group $F_{0} * H$.

Remark 4 . Note that by sending $s$ to the generator $1 \in \mathbb{Z} \subset G=K \rtimes_{\mu} \mathbb{Z}$, we obtain a retraction of $\bar{G}$ onto $G$ (with section induced by the inclusion of $K$ into $\bar{K}$ ).

We can now complete the proof. 
Clearly, the natural homomorphism from $H$ into $\bar{G}$ is injective (and is thus injective into $G$ ). Hence if $H$ is not residually finite, then neither is $G$. It remains to show that if $H$ is residually finite, then so is $\bar{G}$ (and thus $G$ ).

Case 1. If $H$ is finitely generated, then the result holds straightaway by (a) and (b) above.

Case 2. Suppose that $H$ is not finitely generated. For any homomorphism $\theta$ from $H$ to a group $H_{\theta}$ we obtain an induced homomorphism from $\bar{G}=\left(F_{0} * H\right) \rtimes_{\bar{\mu}} \mathbb{Z}$ to $\bar{G}_{\theta}=\left(F_{0} * H_{\theta}\right) \rtimes_{\bar{\mu}} \mathbb{Z}$ which acts as $\theta$ on $H$ and acts as the identity on $F_{0}$ and $\mathbb{Z}$. Let $g=\left(w_{0} h_{1} w_{1} \ldots h_{q} w_{q}\right) . n$ be a non-trivial element of $\bar{G}$, where $q \geq 0, h_{1} \ldots h_{q} \in$ $H-\{1\}, w_{1}, \ldots, w_{q-1} \in F_{0}-\{1\}, w_{0}, w_{q} \in F_{0}, n \in \mathbb{Z}$, and if $q$ is 0 , then either $n \neq 0$ or $w_{0}$ is non-trivial. Since residually finite groups are fully residually finite, there is a homomorphism $\tau$ from $H$ onto a finite group $H_{\tau}$ such that $\tau\left(h_{i}\right) \neq 1$ $(i=1, \ldots, q)$. So the image of $g$ in $\bar{G}_{\tau}=\left(F_{0} * H_{\tau}\right) \rtimes_{\bar{\mu}} \mathbb{Z}$ is non-trivial, and then Case 1 applies.

\section{Proof of TheOREM 5}

Lemma 3. Let $C$ be a group which is a retract of a group B. If $B$ has solvable conjugacy (or power conjugacy) problem, then so does $C$.

Proof. By assumption we have maps $B \stackrel{\rho}{\underset{\mu}{\rightleftarrows}} C, \rho \mu=\mathrm{id}_{C}$. Clearly if $c, d \in C$ are conjugate (respectively, power conjugate) in $C$, then $\mu(c), \mu(d)$ are conjugate (respectively, power conjugate) in $B$. Conversely if there exists $b \in B$ such that $b \mu(c) b^{-1}=\mu(d)$ (respectively, $\left.b \mu(c)^{i} b^{-1}=\mu(d)^{j}\right)$, then $\rho(b) c \rho(b)^{-1}=d$ (respectively, $\left.\rho(b) c^{i} \rho(b)^{-1}=d^{j}\right)$. Thus the result follows.

Now it is shown in [7] that infinite cyclic extensions of finitely generated free groups have a solvable conjugacy, and power conjugacy, problem. By Remarks 2

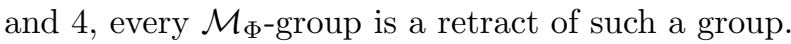

\section{Proof of Theorem 6}

We will assume familiarity with the terminology in $\S \S 1.2,1.4$ of $[6]$.

As in Lemma 1, we can assume that $\theta(x)>0$ for all $x$. We can extend $\theta$ to any word $U=y_{1}^{\varepsilon_{1}} y_{2}^{\varepsilon_{2}} \ldots y_{s}^{\varepsilon_{s}}\left(s>0, y_{i} \in \mathbf{x}, \varepsilon_{i}= \pm 1,1 \leq i \leq s\right)$ by $\theta(U)=\sum_{i=0}^{s} \varepsilon_{i} \theta\left(y_{i}\right)$.

Let $\mathbb{P}$ be a based connected spherical picture (with at least one disc) over $\mathcal{P}$, with global basepoint $O$, and basepoint $O_{\Delta}$ for each disc $\Delta$. (Note that since $R$ is not periodic, there will be just one basepoint for each disc.) We will also choose, for each region $\mathrm{R}$, a point $O_{\mathrm{R}}$ in the interior of $\mathrm{R}$.

We can relabel $\mathbb{P}$ to obtain a picture $\tilde{\mathbb{P}}$ over $\tilde{\mathcal{P}}$ as follows:

(a) For each region $\mathrm{R}$, choose a tranverse path $\gamma_{\mathrm{R}}$ from $O$ to $O_{\mathrm{R}}$, and let $U_{\mathrm{R}}$ (a word on $\mathbf{x}$ ) be the label on the path $\gamma_{\mathrm{R}}$. Then the potential $q(\mathrm{R})$ of $\mathrm{R}$ is $\theta\left(U_{\mathrm{R}}\right)$. (This is independent of the choice of path $\gamma_{\mathrm{R}}$, since $\theta(W)=0$.)

(b) For an arc tranversely labelled $x \in \mathbf{x}$ say, relabel it by $(q(\mathrm{R}), x)$ where $\mathrm{R}$ is the region where the tranverse arrow on the arc begins.

(c) For a corner of a disc, with label $h \in H$ say, relabel the corner by $(q, h)$, where $q$ is the potential of the region in which the corner occurs.

For a disc $\Delta$, let $q_{\Delta}$ be the potential of the region containing $O_{\Delta}$. Then in the relabelled picture, $\Delta$ will be labelled by the path $\left(q_{\Delta}, R\right)$. 
Let $\Theta$ be a minimal disc, that is, a disc such that $q_{\Theta} \leq q_{\Delta}$ for all discs $\Delta$. Let $m$ be the minimum value of $\phi_{W}^{\theta}$, and let $e$ be one of the two distinct letters occurring at the unique minimum. Then in the path $(0, R)$ there is a unique edge labelled $(m, e)$. Now $\Theta$ is labelled by $\left(q_{\Theta}, R\right)$ in $\tilde{\mathbb{P}}$, and thus there is a unique edge labelled $\left(m+q_{\Theta}, e\right)$ incident with $\Theta$. This arc must intersect another disc $\Theta^{\prime}$, which must also be labelled by $\left(q_{\Theta}, R\right)$, but with the opposite orientation. Thus we obtain a dipole in $\tilde{\mathbb{P}}$ where $\Theta, \Theta^{\prime}$ are the discs of the dipole. Reverting to $\mathbb{P}$, this dipole in $\tilde{\mathbb{P}}$ gives rise to a dipole in $\mathbb{P}$.

\section{ACKNOWLEDGEMENT}

I thank the referee for his/her helpful comments.

\section{REFERENCES}

[1] R. B. J. T. Allenby and C. Y. Tang, Residual finiteness of certain 1-relator groups: extensions of results of Gilbert Baumslag, Math. Proc. Camb. Phil. Soc. 97 (1985), 225-230. MR0771817 (86k:20029)

[2] G. Baumslag, Residually finite one-relator groups, Bull. Amer. Math. Soc. 73 (1967), 618620. MR0212078 (35:2953)

[3] G. Baumslag, Free subgroups of certain one-relator groups defined by positive words, Math. Proc. Camb. Phil. Soc. 93 (1985), 247-251. MR691993 (84i:20028)

[4] G. Baumslag and D. Solitar, Some two-generator one-relator non-Hopfian groups, Bull. Amer. Math. Soc. 68 (1962), 199-201. MR0142635 (26:204)

[5] G. Baumslag, A. Miasnikov and V. Shpilrain, Open problems in combinatorial and geometric group theory, http://zebra.sci.ccny.cuny.edu/web/nygtc/problems/

[6] W. A. Bogley and S. J. Pride, Aspherical relative presentations, Proc. Edin. Math. Soc. 35 (1992), 1-39. MR1150949 (93d:57004)

[7] O. Bogopolski, A. Martino, O. Maslakova and E. Ventura, The conjugacy problem is solvable for free-by-cyclic groups, Bull. London Math. Soc. 38 (2006), 787-794. MR2268363

[8] K. S. Brown, Trees, valuations, and the Bieri-Neumann-Strebel invariant, Invent. Math. 90, (1987), 479-504. MR914847 (89e:20060)

[9] V. Egorov, The residual finiteness of certain one-relator groups, Algebraic Systems, Ivanov. Gos. Univ., Ivanovo (1981), 100-121. MR745301 (85i:20035)

[10] J. Howie and S. J. Pride, A spelling theorem for staggered generalized 2-complexes, with applications, Invent. Math. 76 (1984), 55-74. MR739624 (85k:20103)

[11] Kourovka Notebook 15 (2002).

[12] W. Magnus, A. Karrass and D. Solitar, Combinatorial Group Theory (Second Edition), Dover, New York, 1976.

[13] J. Meier, Geometric invariants for Artin groups, Proc. London Math. Soc. (3) 74 (1997), 151-173. MR1416729 (97h:20049)

[14] C. F. Miller III, On group-theoretic decision problems and their classification, Annals of Mathematics Studies 68, Princeton University Press, 1971.

[15] S. Meskin, Nonresidually finite one-relator groups, Trans. Amer. Math. Soc. 164 (1972), 105-114. MR0285589 (44:2807)

[16] B. B. Newman, Some results on one-relator groups, Bull. Amer. Math. Soc. 74 (1968), 568571. MR0222152 (36:5204)

[17] S. J. Pride, Star-complexes, and the dependence problems for hyperbolic complexes, Glasgow Math. J. 30 (1988), 155-170. MR942986 (89k:20049)

[18] J.-P. Serre, Trees, Springer-Verlag, Berlin Heidelberg New York, 1980.

[19] D. Wise, The residual finiteness of positive one-relator groups, Comment. Math. Helv. 76 (2001), 314-338. MR1839349 (2002d:20043)

[20] D. Wise, Residual finiteness of quasi-positive one-relator groups, J. London Math. Soc. (2) 66 (2002), 334-350. MR1920406 (2003f:20043)

Department of Mathematics, University of Glasgow, University Gardens, Glasgow G12 8QW, Scotland, United Kingdom

E-mail address: sjp@maths.gla.ac.uk 